\title{
In vitro study of the effects of reprogramming neonatal rat fibroblasts transfected with TBX18 on spontaneous beating in neonatal rat cardiomyocytes
}

\author{
DAJUN QUAN ${ }^{1-3}$ and $\mathrm{HE} \mathrm{HUANG}^{1-3}$ \\ ${ }^{1}$ Department of Cardiology, Renmin Hospital of Wuhan University; ${ }^{2}$ Cardiovascular Research Institute, \\ Wuhan University; ${ }^{3}$ Hubei Key Laboratory of Cardiology, Wuhan, Hubei 430060, P.R. China
}

Received December 20, 2017; Accepted June 11, 2018

DOI: $10.3892 / \mathrm{mmr} .2018 .9566$

\begin{abstract}
Intracardiac injection of the growth-promoting transcription factor of the sinoatrial node T-box 18 (TBX18) has been shown to reprogram cardiomyocytes into induced sinoatrial nodes that produce high-frequency and neuroregulated ectopic beats. Fibroblasts, the most important non-cardiomyocyte cell type in the heart, can affect the electrophysiological properties of cardiomyocytes by electrically coupling with them. The aim of the present study was to explore the reprogramming of cardiac fibroblasts (CFs) transfected with TBX18 in vitro and observe its effect on the pacing frequency of neonatal rat ventricular cardiomyocytes (NRVMs) in a co-culture system. CFs transfected with TBX18 could be transformed into cardiac myofibroblasts that expressed high levels of hyperpolarization-activated cyclic nucleotide-gated cation channel 4 protein and low levels of connexin 43 (COX43) and COX45 protein. In addition, TBX18-CFs could increase the beating rates of NRVMs and TBX18-NRVMs in a co-culture system. The results of the present study indicated that the TBX18 gene could induce $\mathrm{CFs}$ to undergo a transformation that promotes an increase of the beating rates of NRVMs and TBX18-NRVMs.
\end{abstract}

\section{Introduction}

Reprogramming, a widely used technique in regenerative medicine, is the direct transformation of fully differentiated somatic cell into another type of fully differentiated cell in response to the induction of one or more transcription factors without requiring the induced pluripotent stem cell (iPSC) stage (1). In the laboratory, this technique is generally performed by using fibroblasts as the initial cells to be

Correspondence to: Professor He Huang, Department of Cardiology, Renmin Hospital of Wuhan University, 238 Jiefang Road, Wuhan, Hubei 430060, P.R. China

E-mail: huanghe1977@whu.edu.cn

Key words: biological pacing, fibroblasts, cardiomyocytes, T-box 18, reprogramming reprogramed into induced neurons (2), cardiomyocytes (3) and haematopoietic progenitor cells (4).

Using reprogramming technology, Hu et al (5) successfully reprogrammed T-box 18 (TBX18)-transfected cardiomyocytes directly to induced sinoatrial node (iSAN) cells in adult pig hearts with a complete heart block. Cells in the vicinity of the injection site expressed higher levels of SAN-specific genes and lower levels of chamber-specific genes. TBX18, a transcription factor, is required for the embryonic development of the head area of the SAN but is undetectable after birth and in adulthood (6). In addition, Tbx18 is the only transcription factor that has converted working myocytes into SAN cells and has caused an increase in the spontaneous beating rate (7). In addition, cardiac fibroblasts (CFs), the most important non-cardiomyocyte cell type in the heart, can electrically couple with cardiomyocytes to affect their electrophysiological properties (8). So, it is unclear what changes might occur with fibroblasts when TBX18 is injected directly into the heart and what effect these changes might have on surrounding cardiomyocytes.

In this study, we explored the reprogramming effect of TBX18 on in vitro neonatal rat CF cell cultures and observed the effect of these changes on beating rates when TBX18-CFs were co-cultured with neonatal rat ventricular cardiomyocytes (NRVMs) and TBX18-NRVMs. These data will help us understand the contributions of fibroblasts to the development of biological pacemakers when TBX18 is directly injected into the heart.

\section{Materials and methods}

Materials. Dulbecco's modified Eagle's medium (DMEM)/F12 (1:1) and foetal bovine serum were purchased from Gibco (Thermo Fisher Scientific, Inc., Waltham, MA, USA). Trypsin, type II collagenase and 5-bromodeoxyuridine were purchased from Sigma-Aldrich (Merck KGaA, Darmstadt, Germany). DH5 $\alpha$ competent cells were purchased from Tiangen (Beijing, China). Specific rabbit monoclonal antibodies against hyperpolarization-activated cyclic nucleotide-gated cation channel 4 (HCN4), which is a marker for SAN, connexin 43 (COX43), which is a common connexion between cardiac cells, cardiac troponin I (cTnI), which is a marker for NRVMs, $\alpha$-striated 
actin $(\alpha-\mathrm{SA})$, which is a marker for NRVMs, and GAPDH were purchased from Abcam (Cambridge, MA, USA); antibodies for vimentin, which is a marker for CFs, and COX-45, which is another common connexion between cardiac cells, were purchased from Cell Signaling Technology, Inc. (Danvers, MA, USA) and Santa Cruz Biotechnology, Inc. (Dallas, TX, USA), respectively; and antibodies for myosin heavy chain (MHC), which is a marker for NRVMs, and $\alpha$-striated muscle actin ( $\alpha$-SMA), which is a marker for cardiac myofibroblasts (CMFs) were purchased from Wuhan Sanying Biotechnology (Wuhan, China) and Wuhan Tiandeyue Biotechnology (Wuhan, China), respectively.

Construction of the TBX18 lentiviral vector. pHBAd-MCMV-GFP (Ad-GFP) (Hanbio, Shanghai, China) was digested using Bam HI and NotI. The ORF sequence of the human TBX18 gene (GenScript, Nanjing, China) was amplified using polymerase chain reaction (PCR). After the restriction enzyme digestion, gel extraction was performed. The digested fragment and vector were ligated to form pHBAd-MCMV-GFP-TBX18 (Ad-TBX18), which was then transformed into competent DH5 $\alpha$ cells (Tiangen). Positive clones were identified by liquid sequencing. Bacteria in the logarithmic growth phase were incubated at $37^{\circ} \mathrm{C}$ overnight in LB culture medium while shaking at $300 \mathrm{x}$ g. The large-scale preparation of recombinant plasmids was conducted using a Plasmid Midi Preparation kit (Beijing CW Biotech Co., Ltd., Beijing, China). Cells were transfected with Ad-TBX18 and the backbone vector pHBAd-BHG using Lipofilter ${ }^{\mathrm{TM}}$ (both from Hanbio). The supernatant was harvested after viral amplification. Ad-GFP and Ad-TBX18 were adjusted to $1 \times 10^{10} \mathrm{PFU} / \mathrm{ml}$ and stored at $-80^{\circ} \mathrm{C}$.

Isolation and culture of CFs and NRVMs. The present study was approved by the Ethical Board of The Renmin Hospital of Wuhan University (Wuhan, China). Hearts were dissected from 16 neonatal (1- to 2-day-old) rat heart ventricles, minced and washed in solution A $(0.02 \%$ phenol red, $137 \mathrm{mM} \mathrm{NaCl}$, $5.4 \mathrm{mM} \mathrm{KCl}, 0.34 \mathrm{mM} \mathrm{Na}_{2} \mathrm{HPO}_{4}, 0.44 \mathrm{mM} \mathrm{KH} \mathrm{PO}_{4}, 5.6 \mathrm{mM}$ D-glucose and $20 \mathrm{mM}$ HEPES, $\mathrm{pH}$ 7.3) at room temperature. The heart tissue was dissociated using a digestion solution containing $450 \mathrm{U}$ collagenase and $14 \mathrm{U}$ DNase per ml of solution $\mathrm{A}$ in an Erlenmeyer flask containing glass beads. Then, the flask was placed in a shaking water bath at $37^{\circ} \mathrm{C}$. We pooled cell suspensions from two dissociations and centrifuged the mixture $1,000 \mathrm{x} g$ for $15 \mathrm{~min}$. We then resuspended the cells in Ham's F10 medium supplemented with $10 \%$ fetal bovine serum (FBS) and 10\% horse serum (HS) and plated the cells onto 12 Primaria-coated $6 \mathrm{~cm}$ culture dishes. The cells were plated for $45 \mathrm{~min}$ to allow fibroblasts to preferentially attach to the bottom of the culture dishes. The non-adherent cells (cardiomyocytes) were collected, and the adherent cells (mainly fibroblasts) were supplemented with DMEM containing 10\% FBS and antibiotics (100 U/ml penicillin and $0.1 \mathrm{~g} / 1$ streptomycin). Fibroblasts were grown to confluence and then passaged and plated onto 10 collagen I-coated 6-well stretch plates. The collected cardiomyocytes were directly plated at a density of $1 \times 10^{5}$ cells $/ \mathrm{cm}^{2}$ onto 8 collagen I-coated 6 -well stretch plates and cultured in Ham's F10 and DMEM (1:1) supplemented with $8 \%$ HS and antibiotics. The cardiomyocytes and fibroblasts were incubated at $37^{\circ} \mathrm{C}$ in $5 \% \mathrm{CO}_{2}$ in a humidified incubator. The culture medium used to incubate both cell types was refreshed every 2-3 days.

CFs and NRVMs transfected with TBX18. CFs in passages 3-5 were removed from the culture dishes by digestion. A cell suspension was prepared and inoculated onto 6-well plates. When cell confluence reached 70-80\%, Ad-TBX18 in DMEM/F12 was added to the cells at a multiplicity of infection (MOI) of 100, and these cells were used as the experimental group. CFs that were treated with Ad-GFP were used as the control group. After a 2-h incubation period, the medium was replaced with fresh complete medium. Following the same method, we transfected NRVMs with Ad-TBX18 at an MOI of 100. After a 2-h incubation in an incubator, the medium was replaced, and the cells were cultured for an additional 2 to 5 days. The cells were observed under light and fluorescence microscopy. The percentage of green fluorescent protein-positive cells was determined using flow cytometry (BD Biosciences, Franklin Lakes, NJ, USA).

Western blot assays. TBX18-CFs and GFP-CFs were seeded into 6-well culture dishes. The cells were harvested using RIPA lysis buffer. Equal amounts of protein were loaded onto a gel for sodium dodecyl sulphate-polyacrylamide gel electrophoresis (SDS-PAGE), and the proteins were separated and transferred to nitrocellulose membranes. Then, the membranes were incubated with primary antibodies against HCN4, $\alpha$-SMA, COX43, COX45, cTnI, $\alpha$-SMA and MHC overnight at $4^{\circ} \mathrm{C}$. The bound primary antibodies were detected by incubating the membranes with horseradish peroxidase (HRP)-conjugated secondary antibodies that were raised in the appropriate species, and the results were then detected using enhanced chemiluminescence. The level of GAPDH was used to normalize the signal intensities.

Immunofluorescence staining. After the cells were pre-plated, we calculated the purity of the obtained cell populations, and the CFs and NRVMs were cultured on gelatine-coated coverslips in 6-well culture dishes. The cells were washed with phosphate-buffered saline (PBS) and fixed with $4 \%$ paraformaldehyde. Following permeabilization with $0.1 \%$ Triton X-100, the CFs and NRVMs were incubated with primary anti-vimentin and anti-cTnI antibodies overnight at $4^{\circ} \mathrm{C}$. Secondary antibodies (HRP-Goat anti-Rat IgG and HRP-Goat anti-Rabbit IgG) were then used to detect cTnI. We used 4',6-diamidino-2-phenylindole (DAPI) to visualize the nuclei. The cells were observed under a fluorescence microscope. We randomly selected three visual fields in each of three different cell isolates to calculate the percentage of fluorescent cells in the total number of cells, and the mean value was determined. In addition, the same method was used to incubate TBX18-CFs and GFP-CFs with antibodies against HCN4, vimentin and $\alpha$-SMA to observe expression of these proteins.

Co-culture conditions. In the co-culture experiments, CFs and NRCMs were mixed and plated at a ratio of 1:4 (20\% CFs) on $60-\mathrm{cm}^{2}$ culture dishes. Two groups of co-cultures were established according to the experimental strategy, as follows. The 
first group consisted of co-cultures of NRVMs, NRVMs+CFs, NRVMs+GFP-CFs and NRVMs+TBX18-CFs; the second group consisted of co-cultures of NRVMs, TBX18-NRVMs, TBX18-NRVMs+CFs, TBX18-NRVMs+GFP-CFs and TBX18-NRVMs+TBX18-CFs. The combinations in the first group were co-cultured for 14 days as isotropic monolayers, and the medium was changed after $24 \mathrm{~h}$. Fluorescence mapping was performed after 2 days, the cells were observed once every two days, and the beating frequency was determined in each group by observing red fluorescence using a BX41 microscope. In addition, the mean, min and max number of spontaneous beats were determined in the second group at the time of the highest value observed in the first group. The co-culture experiments were performed three times to validate the results.

Statistical analysis. All data are presented as the mean \pm standard error of the mean. Statistical comparisons among multiple groups were analysed using one-way analysis of variance with Dennett's T3 test in SPSS 19.0 software (IBM Corp., Armonk, NY, USA). $\mathrm{P}<0.05$ was considered to indicate a statistically significant difference.

\section{Results}

Changes in FCs following TBX18 transfection-induced reprogramming

Protein expression in TBX18-CFs was characteristic of iSAN cells. After the cells were pre-plated, we obtained CFs with a purity of $96 \%$. The transfection efficiency in the CFs was $76.2 \pm 6.2 \%$ at an MOI of 100 .

On the 2nd day after cells were transfected with Ad-TBX18, a few cells became round (indicative of dead cells), but the remaining cells were morphologically radial and flaky. There were vacuoles in the cytoplasm of some cells, and cell proliferation slowed down. No spontaneous beating was observed in any of the cultures. After 4-6 days of culture, the CFs proliferated, and their synapses were partially extended without fusiform, triangular or conical changes, which are the morphological characteristics of SANs. In addition, spontaneous beatings were not observed until the 8th day of culture (Fig. 1). These results indicate that the TBX18-CFs did not transform into iSAN cells.

HCN4 is an important channel protein that functions as a pacemaker current (If) channel and is highly expressed in SAN cells (3). The HCN4 protein was expressed at significantly higher levels in TBX18-CFs but was rarely observed in GFP-CFs, which perhaps reflects the immature state of fibroblasts in neonatal rats. The expression levels of COX43 and COX45 were higher in GFP-CFs than in TBX18-CFs $(\mathrm{P}<0.05)$, and the expression levels of $\mathrm{COX}$ proteins were lower in TBX18-CFs than in GFP-CFs (Fig. 2), indicating that TBX18 reduced the connections between CFs.

TBX18-CFs were not converted into cardiomyocytes. cTnI, $\alpha-\mathrm{SA}$ and $\mathrm{MHC}$ are cardiomyocyte-specific proteins. All three proteins were expressed at much lower levels in TBX18-CFs and GFP-CFs than in NRVMs, which were used as the positive control group. In addition, the difference in cTn1 expression was much more conspicuous in each group (Fig. 3). These results indicate that $\mathrm{CFs}$ did not exhibit cardiomyocyte-like phenotypes after they were transfected with TBX18.

TBX18-CFs were transformed into CMFs. Vimentin is a fibroblast-specific protein. Our results showed that under fluorescence microscopy, TBX18-CFs and GFP-CFs exhibited red fluorescence and a filamentous arrangement in the cytoplasm, indicating that these two groups of cells expressed a fibroblast-specific protein and maintained the histological characteristics of fibroblasts (Fig. 4A and B). $\alpha$-SMA is a CMF-specific protein (8). Western blot and immunofluorescence analyses showed that $\alpha$-SMA was expressed at higher levels in TBX18-CFs but at much lower levels in GFP-CFs and CFs, both of which served as negative controls (Fig. 4), indicating that TBX18 induced fibroblasts to transform into CMFs.

TBX18-transfected CFs improved the pulsation rate of co-cultured NRVMs. Spontaneous beating was analysed in the groups of co-cultured cells for 14 days, and the results are shown in Fig. 5A. On the 8th day of co-culture, the rate of spontaneous beating in the NRVM+TBX18-CF cultures was higher ( $86 \pm 10$ b.p.m.) than the rate in the NRVM culture $(65 \pm 9$ b.p.m.) and significantly higher than the rate in the NRVM+GFP-CF and NRVM+CF cultures ( $24 \pm 7$ and $21 \pm 6$ b.p.m., respectively). There was no difference in the rate of spontaneous beating between the NRVM+GFP-CF and NRVM+CF cultures. Spontaneous beating began to decrease in the NRVMs after 8 days in co-culture and then gradually slowed until it stopped on the 14th day.

TBX18-transfected CFs improved spontaneous beating in co-cultured NRVMs that were transfected with TBX18. On the 8th day of co-culture, spontaneous beating was observed in each of the groups (Fig. 5B-D). The cells in the TBX18-NRVM group were beating significantly faster than the cells in the NRVM group (94.20 17.38 vs. $62.10 \pm 11.67$ b.p.m., respectively; $\mathrm{P}<0.001)$, indicating that TBX18 significantly improved myocardial cell pulsation. There was no significant difference in the rates between the CF and GFP-CF co-culture groups $(\mathrm{P}=0.71)$, suggesting that transfecting GFP did not affect spontaneous beating in the co-culture system. There was also no significant difference between the $\mathrm{CF}$ co-culture group and the TBX18-NRVM group $(\mathrm{P}=0.52)$, but co-culturing cells with CFs tended to decrease the spontaneous beating rates. The rate of spontaneous beating in the TBX18-NRVMs was

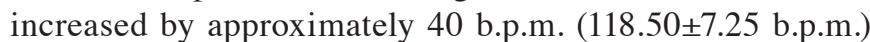
and reached as high as 132 b.p.m. in the TBX18-NRVMs that were co-cultured with TBX18-CFs. These results indicate that TBX18 could abolish the inhibitory effect of CFs on the co-culture system.

\section{Discussion}

In this study, TBX18-CFs were not transformed into iSAN cells, but the protein expression levels of HCN4, COX43 and COX45 were increased. TBX18-CFs also did not undergo a cardiomyocyte-like transformation, but they overexpressed $\alpha$-SMA, indicating that they had transformed into CMFs. In addition, TBX18-CFs could gradually increase the rate of spontaneous beating when they were co-cultured with 

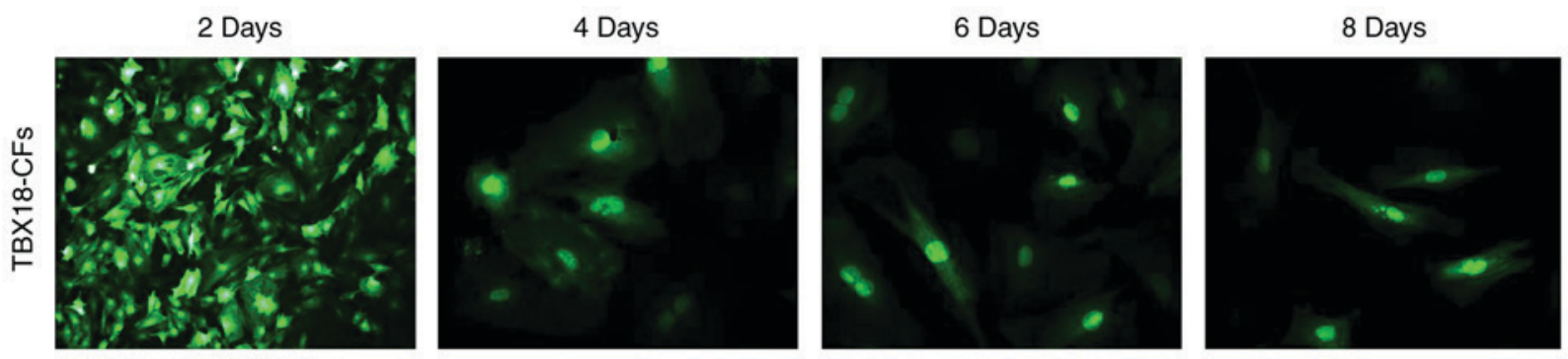

Figure 1. Morphological differences in fibroblasts transfected with TBX18. The morphological changes and pulsatility of CFs were observed by fluorescence microscopy from 2 to 8 days following TBX18 transfection. Cultivated to 2 days (magnification, x100); 4 days (magnification, x200); 6 days (magnification, $\mathrm{x} 200$ ); and to 8 days (magnification, x200). TBX18, T-box18; CFs, cardiac fibroblasts.
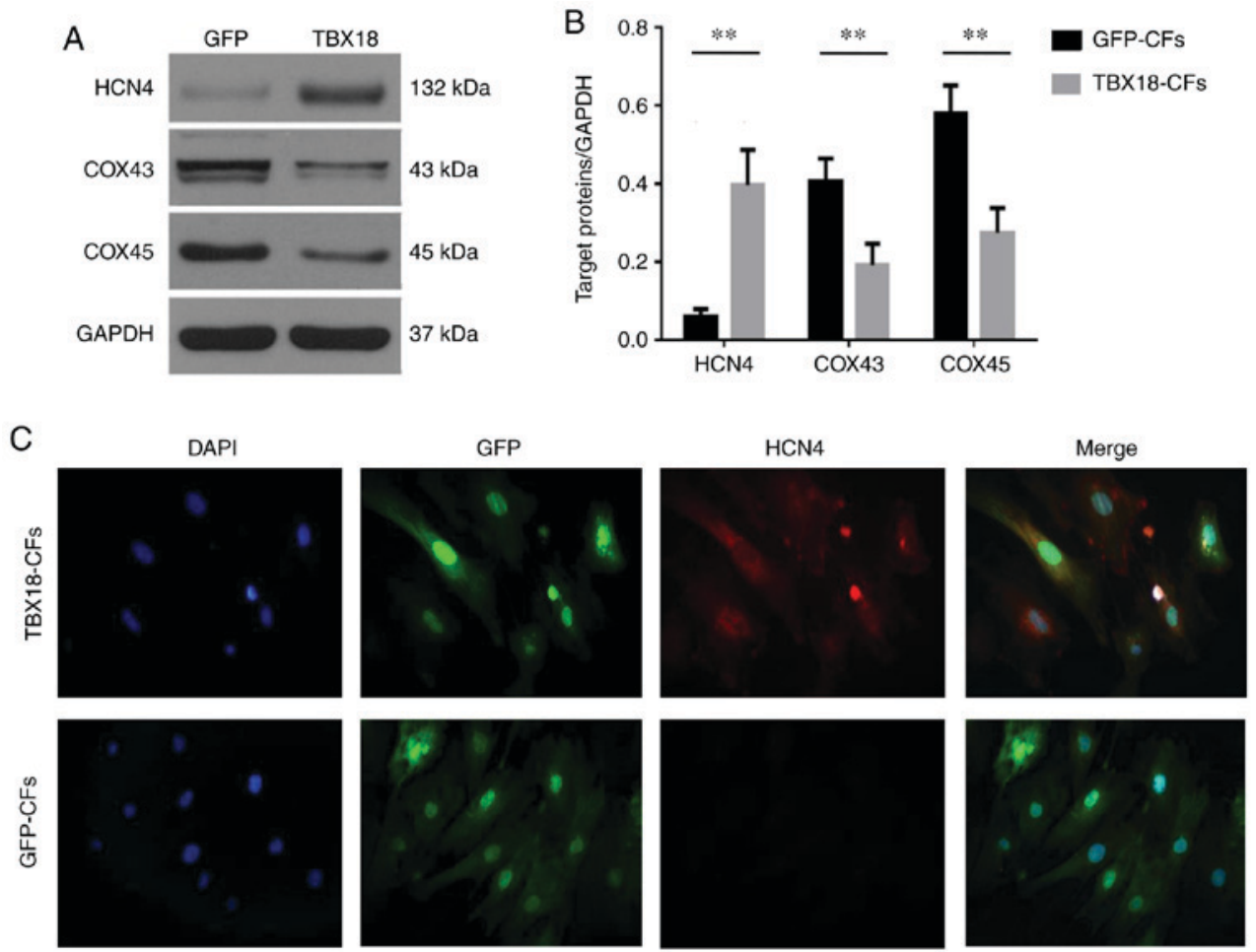

Figure 2. Expression of sinoatrial node specific proteins in fibroblasts transfected with TBX18. (A) The protein expression levels of HCN4, COX43 and COX45 were observed in TBX18-CFs and GFP-CFs. (B) The expression of HCN4 protein in TBX18-CFs was significantly increased; however, the expression levels of COX43 and COX45 were decreased. (C) The expression of HCN4 protein was observed in the TBX18-CF and GFP-CFs groups (magnification, x200). Blue fluorescence indicates nuclear staining, green fluorescence indicates GFP staining and red fluorescence indicates HCN4 protein. ${ }^{* *} \mathrm{P}<0.01$, as indicated. TBX18, T-box18; CFs, cardiac fibroblasts; HCN4, hyperpolarization-activated cyclic nucleotide-gated cation channel 4; COX, connexin; GFP, green fluorescence protein.

NRVMs or TBX18-NRVMs. However, GFP-transfected CFs and CFs showed the opposite effect, indicating that the TBX18 gene could induce CFs to transform, which contributed to the increase of the beating rates of NRVMs and TBX18-NRVMs.

In 2006, Takahashi and Yamanaka et al (9) found that multiple transcription factors can be combined to reverse the embryonic fibroblasts into iPSCs; these iPSCs can be transformed into other cells if exposed to a certain inductive environment. However, many studies have found that transfecting certain single transcription factors could cause cells to undergo cross-line reprogramming. For example, myogenic differentiation (MyoD) can directly reprogram fibroblasts into skeletal muscle cells (10). The transcription factor octamer-binding factor 4 (OCT4) directly reprogrammed blood cells into induced neural progenitor cell (iNPCs) (11) without requiring the intermediate step of forming iPSCs. This means the technology to directly transform one cell into another cell is possible, which would require a short cycle and a simple process and potentially reduce teratoma formation and the risk of mutation (12). Direct reprogramming has also been used in biological pacing. Kapoor et al (7) found that cardiomyocytes induced by TBX18 could directly transform them into pacing iSAN cells both in vivo and in vitro. Hu et al (5) reported similar results in large animal experiments. Transfecting TBX18 into neonatal rat hearts could decrease the expression of COX43, increase the expression of inward rectifier potassium channels and HCN4, and then increase the spontaneous beating rates of heart (7). Additionally, transfection with TBX18 could increase the regulation of cAMP (e.g., the 'membrane clock' and ' $\mathrm{Ca}+$ clock') and induced spontaneous local Ca2+ release (LCR) events (4). These data indicate that TBX18-reprogrammed cardiomyocytes exhibit stable pacing functions. 

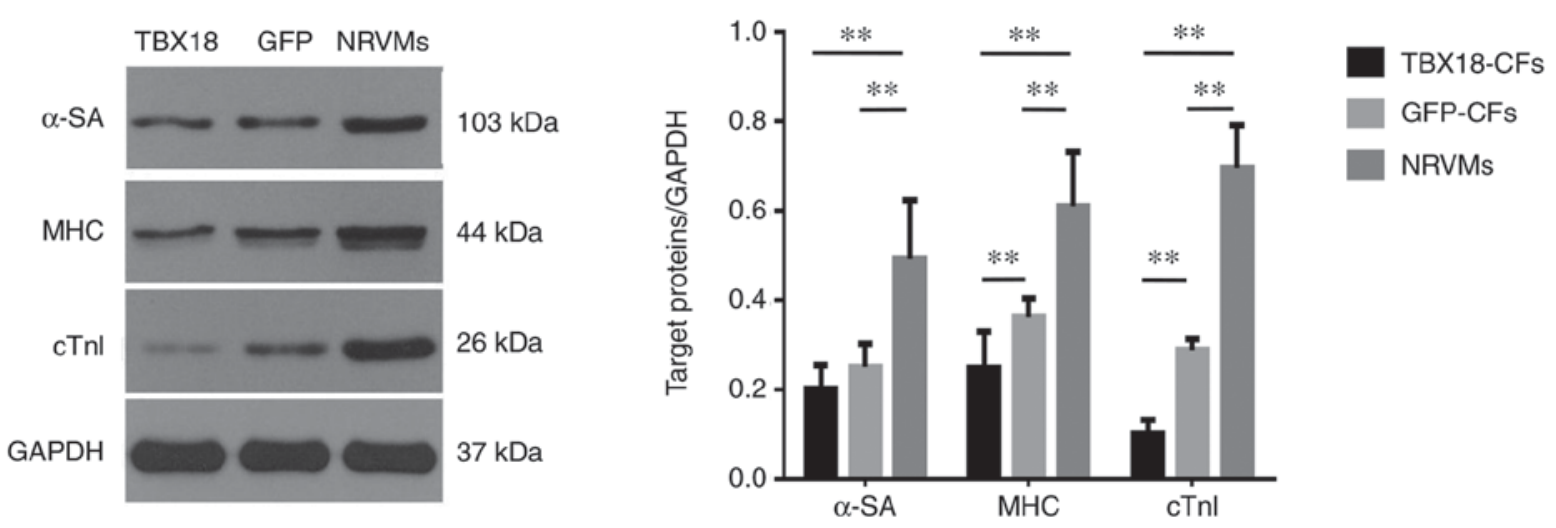

Figure 3. Expression of cardiomyocyte-specific proteins in fibroblasts transfected with TBX18. The expression of $\alpha$-SA, MHC and cTnI was observed in transfected fibroblasts and compared with that in NRVMs. None of the three proteins were observed in fibroblasts transfected with TBX18. ${ }^{* *} \mathrm{P}<0.01$, as indicated. TBX18, T-box18; CFs, cardiac fibroblasts; NRVMs, neonatal rat ventricular cardiomyocytes; cTnI, cardiac troponin I; $\alpha$-SA, $\alpha$-striated actin; MHC, myosin heavy chain.
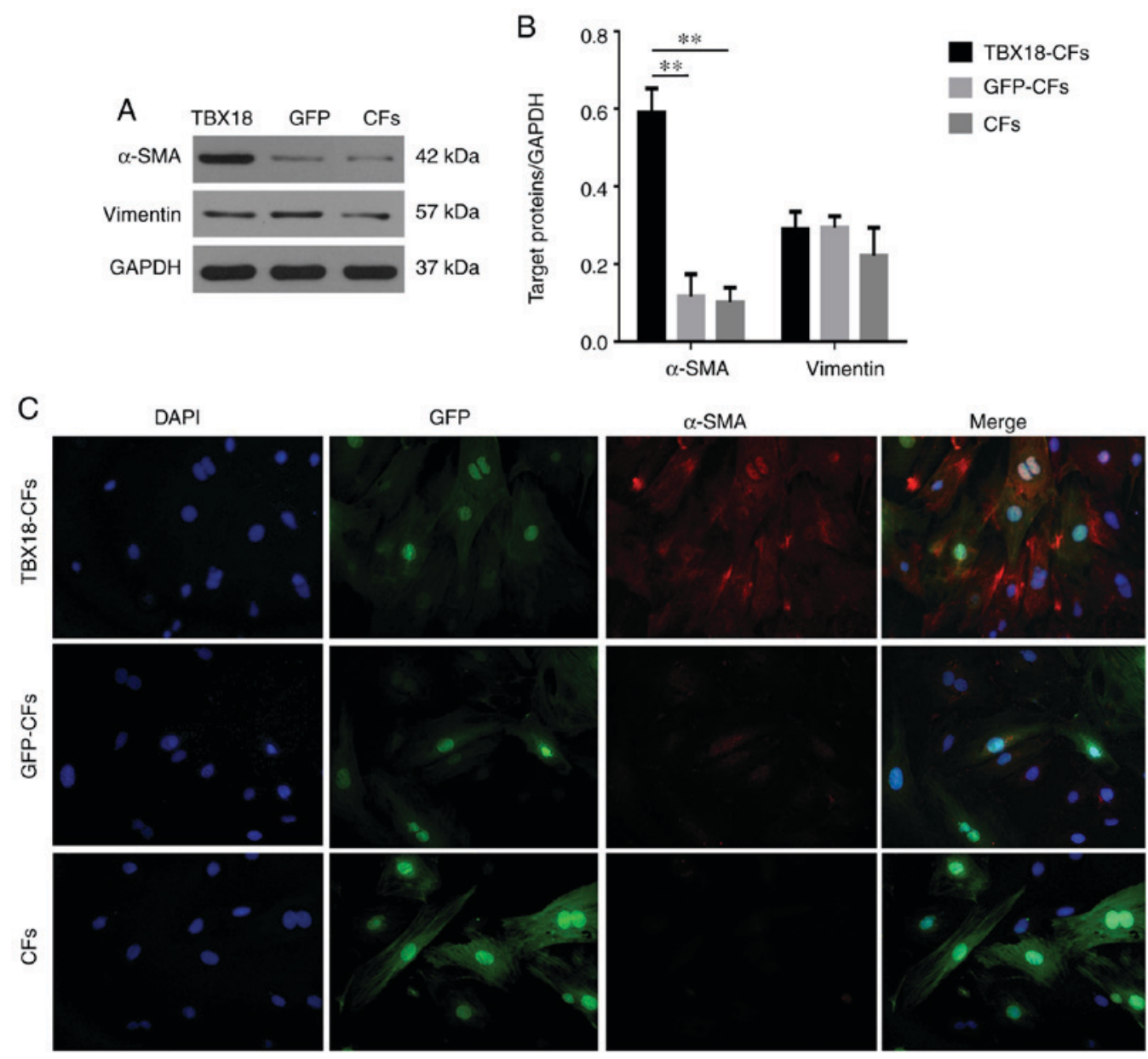

Figure 4. Expression of $\alpha$-SMA and Vimentin in fibroblasts transfected with TBX18. (A) The expression of $\alpha$-SMA and Vimentin in TBX18-CFs, GFP-CFs and CFs was (B) compared with that observed in non-transfected fibroblasts. The expression of $\alpha$-SMA protein in TBX18-CFs was significantly increased, while Vimentin expression showed no difference. (C) Fluorescence microscopy was used to observe the expression of $\alpha$-SMA protein (magnification, x200). Blue fluorescence indicates nuclear staining, green fluorescence indicates GFP staining and red fluorescence indicates $\alpha$-SMA protein. ${ }^{* *} \mathrm{P}<0.01$, as indicated. TBX18, T-box18; CFs, cardiac fibroblasts; $\alpha$-SMA, $\alpha$-smooth muscle actin; GFP, green fluorescence protein.

Fibroblasts, as the most numerous non-cardiomyocyte cell type in heart, account for $45 \%$ of the total number of cardiac cells (13). Under physiological conditions, fibroblasts continue to synthesize and degrade both collagen and fibronectin to support the structural integrity of the heart. Fibroblasts can also directly influence the electrophysiological activity of the myocardium by forming gap junctions with myocardium (14). Fibroblasts are non-excitatory cells that have high membrane resistance and high resting potential and can produce mechanically sensitive currents (8). Therefore, we can predict that 

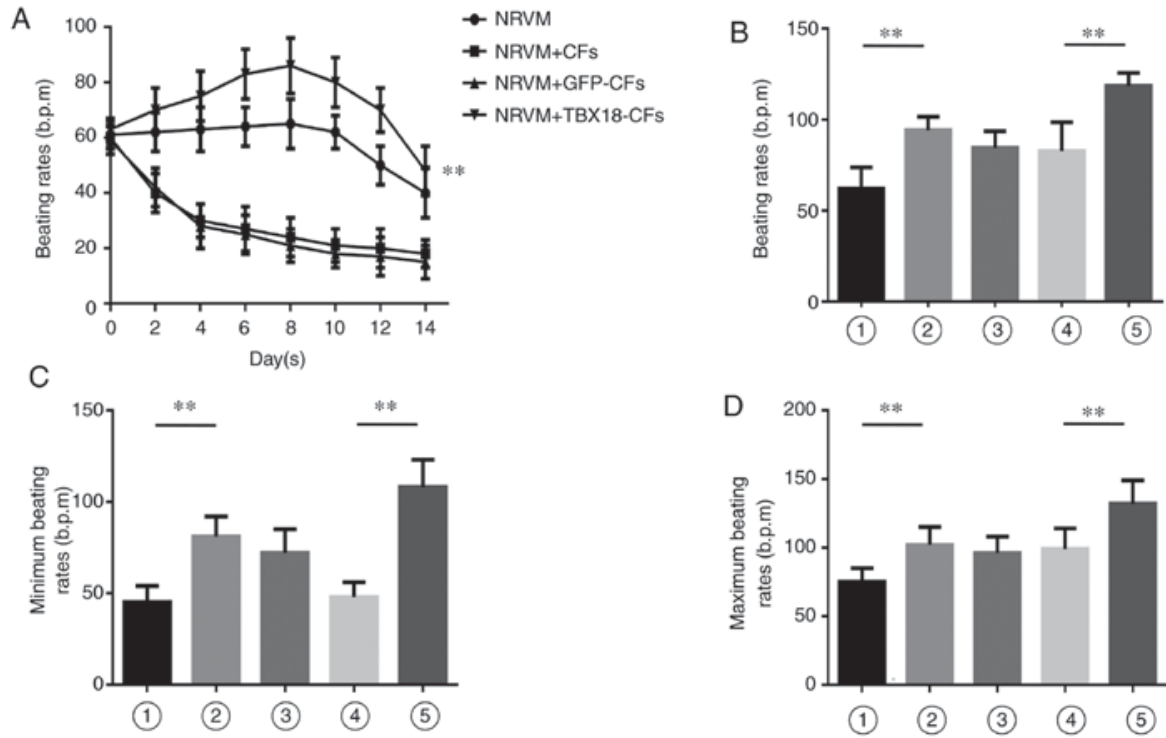

Figure 5. Differences in pulsation frequency were observed in the co-culture system. (A) TBX18-CFs improved the beating rate of NRVMs in the co-culture system. The frequency of spontaneous beating of TBX18-CFs+NRVMs was significantly higher than that of the other groups. The (B) beating rates, and the (C) minimum and (D) maximum beating rates were compared in each group. The beating frequency of TBX18-NRVMs increased by $\sim 40$ to 132 bpm (depending on the conditions) when TBX18-NRVMs were co-cultured with TBX18-CFs. Bar 1, NRVMs; bar 2, TBX18-NRVMs; bar 3, TBX18-NRVMs+CFs; bar 4, TBX18-NRVMs+GFP-CFs; and bar 5, TBX18-NRVMs+TBX18-CFs. ${ }^{* *}$ P $<0.01$, as indicated. TBX18, T-box18; CFs, cardiac fibroblasts; NRVMs, neonatal rat ventricular cardiomyocytes; GFP, green fluorescence protein; bpm, beats per minute.

when the TBX18 gene is injected into the heart of animals, both cardiomyocytes and fibroblasts could be transfected. However, what type of alterations of the characteristics of CFs and the effect of these alterations to adjacent cardiomyocytes has never been studied. Here, we observed that TBX18-CFs could increase the beating rates of NRVMs or TBX18-NRVMs in a co-culture system, but GFP-CFs and CFs showed the opposite effect. Previous research indicates that COX43 is mainly expressed between the myocardium, while COX45 is mainly expressed in fibroblasts (15), and fibroblasts in SANs form electrical couplings and exchange materials with P cells via COX45 (14). In vitro co-cultures of fibroblasts and cardiomyocytes with an increased density of fibroblasts exhibited decreased beating rates of the cardiomyocytes (16), but when COX43 was inhibited in the fibroblasts, the pulse and conduction rate were significantly restored $(17,18)$, indicating that when fibroblasts and cardiomyocytes are adjacent to each other, the higher resting potential of the fibroblasts induces peripheral myocardial cell depolarization through COX43, which reduces their excitability (19). In this study, we observed that the expression of both COX43 and COX45 was decreased in TBX18-CFs. Compared with the results of Hu's research (5), our data show a significant decrease in COX43 expression (which is similar with Hu's results), but COX45 expression just decreased to physiologically normal levels and was still higher than COX43 expression. Therefore, we hypothesised that the TBX18 gene removes the restraint of CFs on NRVMs by decreasing COX43 expression and then delivering an inward current (which is generated by HCN4) to adjacent NRVMs via COX45, therefore increasing the beating rates of NRVMs in the co-culture system.

At the same time, several researchers have found that TBX18 can reprogram cardiomyocytes into iSANs $(5,7)$, but according to our study, TBX18 cannot induce the same activity in CFs, which were simply transformed to CMFs with the protein properties of SANs. Therefore, we consider that the effects of TBX18 on CFs and cardiomyocytes are different. Though many studies have found that CFs could be directly programmed into other types of cells (2-4), our study considers that TBX18 alone was insufficient to reprogram $\mathrm{CFs}$, but it could transform them into similar cells (CMFs), which are common in heart and belong to same family as iSANs.

There are some limitations in this paper that should be noted. First, we used neonatal fibroblasts and cardiomyocytes, which exhibit phenotypes that are not similar to either mature cardiomyocytes or mature nodal cells. The effect of TBX18 on neonatal and mature cells may therefore not be the same as its effect in mature cells. This limits speculation regarding the possibility of reprogramming cardiomyocytes and fibroblasts using TBX18 in adult hearts. Second, because of limitations associated with the experiment environment, no functional studies were performed (e.g., an evaluation of ion channels or fura uptake) apart from an analysis of the beating rate. We therefore could not determine whether transfected CFs might potentially produce arrhythmia or other adverse reactions. Further research should be performed to evaluate these possibilities in the future.

\section{Acknowledgements}

The authors would like to thank Hubei Key Laboratory of Cardiology, Renmin Hospital of Wuhan University (Wuhan, China) for providing experimental materials and support. The authors are also grateful to Dr Yu Liu (Department of Cardiology, Renmin Hospital of Wuhan University) for making detailed adjustments of the procedures during the experiments. 


\section{Funding}

The present study was supported by a grant from the National Natural Science Foundation of China (grant no. 81570306).

\section{Availability of data and materials}

The datasets used and/or analyzed during the current study are available from the corresponding author on reasonable request.

\section{Authors' contributions}

HH conceived the study and DJQ performed the experiments.

\section{Ethics approval and consent to participate}

The present study was approved by the Ethical Board of The Renmin Hospital of Wuhan University (Hubei, China).

\section{Patient consent for publication}

Not applicable.

\section{Competing interests}

The authors declare that they have no competing interests.

\section{References}

1. Zhou Q and Melton DA: Extreme makeover: Converting one cell into another. Cell Stem Cell 3: 382-388, 2008.

2. Vierbuchen T, Ostermeier A, Pang ZP, Kokubu Y, Südhof TC and Wernig M: Direct conversion of fibroblasts to functional neurons by defined factors. Nature 463: 1035-1041, 2010.

3. Ieda M, Fu JD, Delgado-Olguin P, Vedantham V, Hayashi Y, Bruneau BG and Srivastava D: Direct reprogramming of fibroblasts into functional cardiomyocytes by defined factors. Cell 142: 375-386, 2010.

4. Szabo E, Rampalli S, Risueño RM, Schnerch A, Mitchell R, Fiebig-Comyn A, Levadoux-Martin M and Bhatia M: Direct conversion of human fibroblasts to multilineage blood progenitors. Nature 468: 521-526, 2010.

5. Hu YF, Dawkins JF, Cho HC, Marbán E and Cingolani E: Biological pacemaker created by minimally invasive somatic reprogramming in pigs with complete heart block. Sci Transl Med 6: 245ra94, 2014.
6. Wiese C, Grieskamp T, Airik R, Mommersteeg MT, Gardiwal A, de Gier-de Vries C, Schuster-Gossler K, Moorman AF, Kispert A and Christoffels VM: Formation of the sinus node head and differentiation of sinus node myocardium are independently regulated by Tbx18 and Tbx3. Circ Res 104: 388-397, 2009.

7. Kapoor N, Liang W, Marbán E and Cho HC: Direct conversion of quiescent cardiomyocytes to pacemaker cells by expression of Tbx18. Nat Biotechnol 31: 54-62, 2013.

8. Ongstad E and Kohl P: Fibroblast-myocyte coupling in the heart: Potential relevance for therapeutic interventions. J Mol Cell Cardiol 91: 238-246, 2016.

9. Takahashi $\mathrm{K}$ and Yamanaka S: Induction of pluripotent stem cells from mouse embryonic and adult fibroblast cultures by defined factors. Cell 126: 663-676, 2006.

10. Weintraub H, Tapscott SJ, Davis RL, Thayer MJ, Adam MA, Lassar AB and Miller AD: Activation of muscle-specific genes in pigment, nerve, fat, liver, and fibroblast cell lines by forced expression of MyoD. Proc Natl Acad Sci USA 86: 5434-5438, 1989.

11. Halder G, Callaerts P and Gehring WJ: Induction of ectopic eyes by targeted expression of the eyeless gene in Drosophila. Science 267: 1788-1792, 1995.

12. Chambers SM and Studer L: Cell fate plug and play: Direct reprogramming and induced pluripotency. Cell 145: 827-830, 2011.

13. Brown TR, Krogh-Madsen T and Christini DJ: Computational approaches to understanding the role of fibroblast-myocyte interactions in cardiac arrhythmogenesis. Biomed Res Int 2015: 465714, 2015

14. Goldsmith EC, Hoffman A, Morales MO, Potts JD, Price RL, McFadden A, Rice M and Borg TK: Organization of fibroblasts in the heart. Dev Dyn 230: 787-794, 2004.

15. Kizana E, Ginn SL, Allen DG, Ross DL and Alexander IE: Fibroblasts can be genetically modified to produce excitable cells capable of electrical coupling. Circulation 111: 394-398, 2005.

16. Rosker C, Salvarani N, Schmutz S, Grand T and Rohr S: Abolishing myofibroblast arrhythmogeneicity by pharmacological ablation of $\alpha$-smooth muscle actin containing stress fibers. Circ Res 109: 1120-1131, 2011.

17. Haraguchi Y, Shimizu T, Yamato M and Okano T: Electrical interaction between cardiomyocyte sheets separated by non-cardiomyocyte sheets in heterogeneous tissues. J Tissue Eng Regen Med 4: 291-299, 2010.

18. Fahrenbach JP, Mejia-Alvarez R and Banach K: The relevance of non-excitable cells for cardiac pacemaker function. J Physiol 585: 565-578, 2007.

19. Abramochkin DV, Lozinsky IT and Kamkin A: Influence of mechanical stress on fibroblast-myocyte interactions in mammalian heart. J Mol Cell Cardiol 70: 27-36, 2014.

(7) (3) This work is licensed under a Creative Commons (c) AY No Attribution-NonCommercial-NoDerivatives 4.0 International (CC BY-NC-ND 4.0) License. 